\title{
Dielectric Screening Modulates Semiconductor Nanoplatelet Excitons
}

Ashley J. Shin, ${ }^{1}$ Azmain A. Hossain, ${ }^{1}$ Stephanie M. Tenney, ${ }^{1}$ Xuanheng Tan, ${ }^{1}$ Lauren A. Tan, ${ }^{1}$ Jonathan J. Foley IV, ${ }^{2}$ Timothy L. Atallah ${ }^{1, *}$ and Justin R. Caram ${ }^{1, *}$

${ }^{1}$ Department of Chemistry and Biochemistry, University of California, Los Angeles,

607 Charles E. Young Drive, Los Angeles, California, 90095-1569, United States

${ }^{2}$ Department of Chemistry,

William Paterson University,

300 Pompton Road, Wayne, New Jersey, 07470, United States

\section{Abstract}

The influence of external dielectric environments is well understood for 2D semiconductor materials but is overlooked for colloidally-grown II-VI nanoplatelets (NPLs). In this work, we synthesize MX (M=Cd, Hg; X= Se, Te) NPLs of varying thicknesses, and apply a modified Elliott model to fit excitonic absorption features and report exciton binding energies for cadmium telluride and mercury chalcogenides for the first time. Our observations indicate that the exciton binding energy is modulated by the dielectric screening of semiconductor material by the external ligand environment. Furthermore, NPL binding energies show a dependence on the number of monolayers consistent with relative effect of internal vs. external dielectric. To describe this, we derive an analytical electrostatic model, reinforcing the hypothesis that the external environment increases the exciton binding energy relative to the bulk-due to the distortion of the Coulombic potential across the NPL surface. We further confirm this effect by decreasing and recovering the exciton binding energy of HgTe NPLs through washing in polarizable solvents. Our results illustrate that NPLs are colloidal analogues of Van der Waals 2D semiconductors and point to surface modification as an approach to control photophysics and device properties. 


\section{Introduction}

Colloidal nanoplatelets (NPLs) are an emerging class of optoelectronic substrates, useful due to their high absorptivities, near unity quantum yields, and narrow tunable quantum confined emission. $^{0-4}$ Another appealing aspect of these NPLs is the atomic precision over their growth anisotropy: II-VI cadmium chalcogenide NPLs can be synthesized with precisely 2-11 monolayer (ML) thicknesses, and show increasing control over their 2D lateral extent. ${ }^{5-9}$ Such structures can also template other II-VI NPLs, including core-shell and core-crown $\mathrm{Cd} / \mathrm{Zn} \mathrm{S} / \mathrm{Se} / \mathrm{Te}$ heterostructures and mercury chalcogenide infrared emitters, which demonstrate comparable synthetic control. ${ }^{10-12}$ With increased tunability of the NPL thickness, colloidal 2D materials warrant further exploration of the extent of synthetic modulation over their photophysical

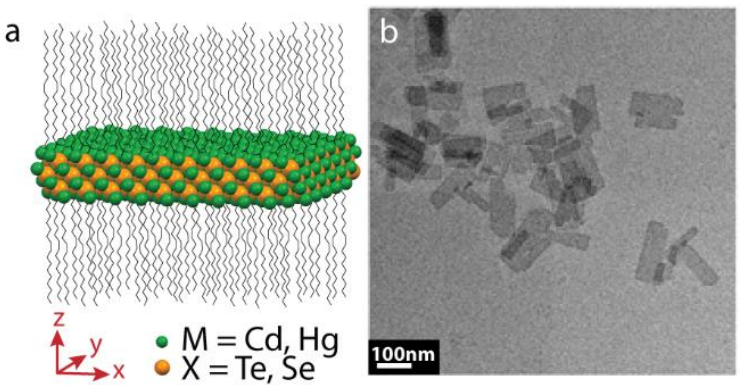

C

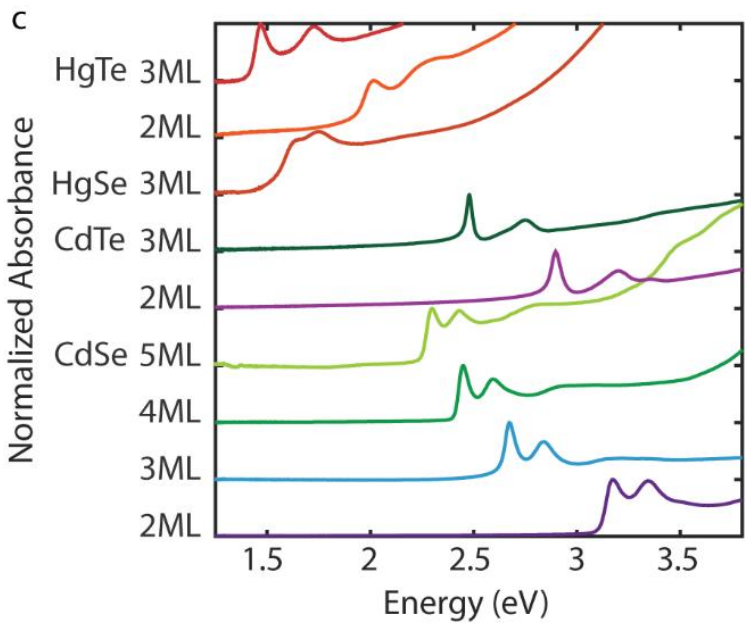

Figure 1. (a) Simplified crystal structure of $3 \mathrm{ML}$ $\mathrm{MX}(\mathrm{M}=\mathrm{Cd}, \mathrm{Hg} ; \mathrm{X}=\mathrm{Se}, \mathrm{Te}) \mathrm{NPL}$, including organic ligands as an example. (b) TEM image of $3 \mathrm{ML}$ HgSe NPLs, showing nanoscale lateral dimensions. (c) Normalized absorbance spectra MX NPLs of varying thickness, offset for clarity. properties and device applicability. In this work, we measure the absorption spectra of zinc-blende II-VI NPLs, which consist of alternating $\mathrm{M}-\mathrm{X}$ atomic layers $(\mathrm{M}=\mathrm{Cd}, \mathrm{Hg}$ and $\mathrm{X}=\mathrm{Se}, \mathrm{Te})$, as shown in Fig. 1a. The NPLs have thicknesses of 0.7-1.9 nm and lateral dimensions of 50-200 nm (Fig. 1b). ${ }^{13-15}$ The synthesis procedures for individual NPLs and material characterization can be found in the Supporting Information (SI Sec. I-II). Motivated by their similarity to other 2D excitonic semiconductors, such as quantum wells and transition metal dichalcogenides (TMDCs), we use the distinct room temperature features of the 
absorption spectra (Fig. 1c) to understand band gap and excitonic properties. In this work, we apply the Elliott model to fit excitonic absorption features. ${ }^{16-19}$ We extract light and heavy hole exciton binding energies $\left(E_{B}\right)$ that are in excellent agreement to the prior limited available experimental results for the heavy hole exciton binding energy in CdSe NPLs. ${ }^{20,21}$ In all NPLs we observe high exciton binding relative to bulk semiconductors.

In layered Van der Waals (VdW) semiconductors, high binding energies are attributed to the strong influence of the external dielectric on $E_{B} \cdot{ }^{22}$ To test whether external dielectric plays a similar role in 2D NPLs we study the thickness and solvent dependence on exciton binding energy. We observe that as NPL thickness increases, exciton binding energy decreases, a result that we can recover quantitatively through a minimal electrostatic model of variable thickness dielectric slabs. We further demonstrate dielectric modulation of binding energy by shifting and recovering HgTe $E_{B}$ through exchange in polarizable solvent. Our results expand upon established colloidal NPLs' excitonic properties and emphasize the importance of the dielectric environment in modulating the photophysical properties.

\section{Results and Discussion}

To establish factors influencing colloidal NPL excitons, we use the absorbance spectra to derive photophysical constants, such as exciton binding energy and band-to-band gap energy $\left(E_{G}\right)$, through Elliott model fits. This straightforward method allows for high-throughput measurements to determine $E_{B}$ values as well as easily monitor solvent dielectric effects on $E_{B}$.

\section{A. Multiband Elliott Model of Colloidal NPL Systems}

The Elliott model describes absorption spectra of 2D and 3D semiconductors, accounting for excitonic resonances and oscillator strengths. The model yields optical transitions, corresponding 
to excitations from the ground state (valence band electrons) to bound electron-hole excitonic states, followed by a higher energy valence-band to conduction-band continuum of free electron transitions. The absorption features depend on the dimensionality of the semi-conductor as shown in Eq. 1:

$$
\begin{gathered}
\alpha_{D}(E) \propto \sum_{n=1}^{\infty}\left(\frac{E_{B} \Gamma(n+D-2)}{(n-1) !\left(n+\frac{D-3}{2}\right)^{D+1}} \cdot \delta\left(E-E_{G}+\frac{E_{B}}{\left(n+\frac{D-3}{2}\right)^{2}}\right)\right) \\
+\frac{\left|\Gamma\left(\frac{D-1}{2}+i \beta\right)\right|^{2} e^{\pi \beta} \beta^{2-D}}{2^{D} \pi^{2-\frac{D}{2}} \Gamma\left(\frac{D}{2}\right)} \cdot \Theta\left(E-E_{G}\right)
\end{gathered}
$$

a
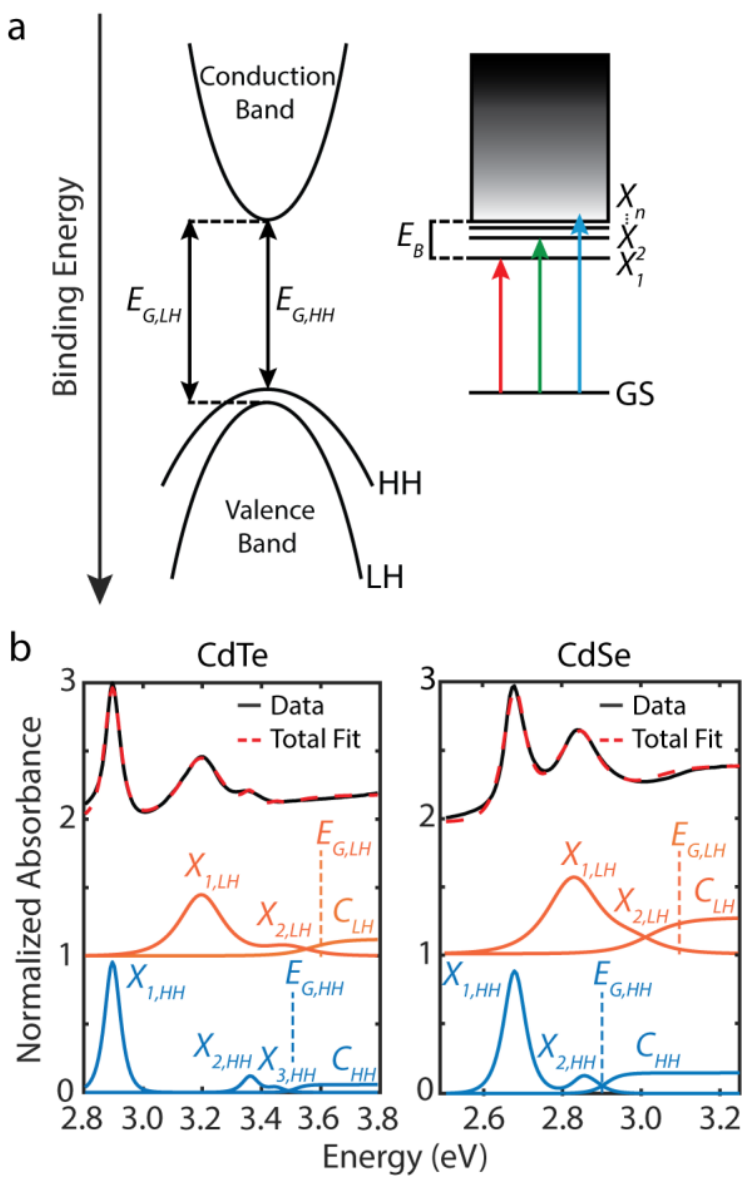

Figure 2. (a) Left: Band diagram, showing both light hole $(\mathrm{LH})$ and heavy hole $(\mathrm{HH})$ valence bands. Right: general transition diagram for a $\mathrm{HH}$ or $\mathrm{LH}$ exciton. (b) Absorption spectra of 2 ML CdTe and 3 ML CdSe NPLs, showing the excitonic features of the modified Elliott model, offset for clarity. The $X n$ transitions and the onset of the conduction band $(C)$ are shown. where $\omega$ is the excitation frequency, $D$ is the dimensionality of the model system, $n$ is the number of discrete excitonic peaks, $\mathrm{E}_{\mathrm{G}}$ is the band gap energy, $\beta=\sqrt{\frac{E_{B}}{\hbar \omega-E_{G}}}, \Gamma(n)=(n-1)$ ! is the gamma function, $\delta(a-b)$ is the Dirac delta function, and $\Theta$ is the Heaviside step function. ${ }^{19,23}$ The model reproduces the intensity and absolute positions of absorption features using only $E_{B}$ and $E_{G}$ as inputs, for a given dimensionality constant $D$. The Elliott model has been used to describe interband transitions, TMDCs, quantum wells, and 2D layered perovskites - but has not been applied to NPLs. ${ }^{16,24-28}$ 
In Fig. 2a, we illustrate the electronic structure of II-VI NPLs and highlight the transitions that are well-described by the Elliott model. The valence band is split into non-degenerate heavy hole (HH) and light hole (LH) bands, which are offset due to spin-orbit coupling. ${ }^{29,30}$ In excitonic materials, the exciton binding energy is greater than thermal energy at room temperature $\left(E_{B}>\right.$ $k T$ ). This results in the observed excitonic transition peaks (Fig. 2a: GS $\rightarrow X_{n}$ ) within the absorption spectrum, appearing at lower energy than their corresponding band-to-band continuum $\mathrm{HH}$ and LH transitions (Fig. 2a: $E_{G, H H}$ and $E_{G, L H}$ ).

We extract the $E_{B}$ and $E_{G}$ values for our II-VI NPLs with various ML thicknesses by fitting our observed absorption spectra with the Elliott model (a more detailed explanation of the fitting procedure can be found in SI Sec. III). To account for the linewidth broadening, we convolve Eq. 1 (where $\mathrm{D}=3$ ) with a tunable hyperbolic secant function $\left(\operatorname{sech}\left(\frac{\hbar \omega}{\gamma}\right)\right)$, resulting in the following expression:

$$
\begin{aligned}
\alpha_{x}(\omega) \propto \sum_{n=1}^{\infty} \frac{2 E_{B}}{n^{3}} \operatorname{sech}\left(\frac{\hbar \omega-E_{G}+\frac{E_{B}}{n^{2}}}{\gamma}\right) \\
\quad+\int_{E_{G}}^{\infty} \operatorname{sech}\left(\frac{\hbar \omega-\tau}{\gamma}\right) \frac{1}{1-\exp \left(-2 \pi \sqrt{\frac{E_{B}}{\hbar \omega^{\prime}-E_{G}}}\right)} d \omega^{\prime}
\end{aligned}
$$

where $\alpha_{X}$ is either $\alpha_{H H}$ or $\alpha_{L H}, \gamma$ is the linewidth broadening factor, and $\hbar \omega^{\prime}$ is the convolution integration energy variable (step-by-step process of the convolution in SI Sec. IV). We observe that the 3D Elliott model generates a better fit than 2D, which we hypothesize is the result of the finite thickness of NPLs. In SI Sec. V, we provide a comparison between the two- and threedimensional Elliott models and fits. We account for $\mathrm{HH}$ and $\mathrm{LH}$ absorption by fitting the sum 
$\alpha_{\text {Total }}=\alpha_{H H}+\alpha_{L H}{ }^{31,32}$ In Fig. 2b, we show examples of typical NPL absorption spectra, the Eq. 2 fit, and fit components corresponding to $\mathrm{HH}$ and $\mathrm{LH}$ transitions. The spectra are broken down into contributions from continuum and excitonic features with labelled transitions. This straightforward application of the Elliott model determines the absolute positions of $\mathrm{HH} / \mathrm{LH}$ excitonic transitions from the onset of the continuum. Thus, the modified Elliott model fits the absorption spectra using only 8 parameters-line-broadening as well as bandgap and exciton binding energies for light and heavy holes and amplitudes.

TABLE I. Bandgap and exciton binding energies extracted from room-temperature absorption data, using a modified Elliott model. The CdX NPLs are passivatd by oleic acid ligands, whereas HgX NPLs are passivated by oleylamine. Both NPLs are suspended in haxane.

\begin{tabular}{lllllll}
\hline \hline NPL & MLs & $\mathrm{E}_{\mathrm{G}, \mathrm{HH}}(\mathrm{eV})$ & $\mathrm{E}_{\mathrm{B}, \mathrm{HH}}(\mathrm{meV})$ & $\mathrm{E}_{\mathrm{G}, \mathrm{LH}}(\mathrm{eV})$ & $\mathrm{E}_{\mathrm{B}, \mathrm{LH}}(\mathrm{meV})$ & Bulk $_{\mathrm{G}}(\mathrm{eV})$ \\
\hline $\mathrm{CdSe}$ & 2 & $3.5 \pm 0.1$ & $430 \pm 30$ & $3.6 \pm 0.1$ & $220 \pm 40$ & $1.7^{\mathrm{c}}$ \\
& 3 & $2.91 \pm 0.05$ & $220 \pm 20$ & $3.1 \pm 0.1$ & $190 \pm 30$ & \\
& & & $210^{\mathrm{a}}$ & & & \\
& 4 & $2.65 \pm 0.05$ & $195 \pm 20$ & $2.7 \pm 0.1$ & $170 \pm 30$ & \\
& & $190^{\mathrm{a}}$ & & & \\
& 5 & $2.45 \pm 0.05$ & $175 \pm 20$ & $2.5 \pm 0.1$ & $120 \pm 30$ & \\
\hline $\mathrm{CdTe}$ & 2 & $3.51 \pm 0.05$ & $620 \pm 30$ & $3.6 \pm 0.1$ & $370 \pm 35$ & $1.54^{\mathrm{d}}$ \\
& 3 & $2.67 \pm 0.05$ & $210 \pm 30$ & $2.9 \pm 0.1$ & $200 \pm 30$ & \\
\hline $\mathrm{HgSe}$ & 3 & $1.92 \pm 0.05$ & $300 \pm 25$ & $1.9 \pm 0.1$ & $190 \pm 35$ & $0^{\mathrm{e}}$ \\
\hline $\mathrm{HgTe}$ & 2 & $2.13 \pm 0.05$ & $450 \pm 30$ & $2.5 \pm 0.1$ & $300 \pm 30$ & $0^{\mathrm{f}}$ \\
& 3 & $1.77 \pm 0.05$ & $350 \pm 25$ & $1.9 \pm 0.1$ & $260 \pm 30$ & \\
\hline
\end{tabular}

${ }^{\mathrm{a}}$ Ref. 20, ${ }^{\mathrm{b}}$ Ref. 21, ${ }^{\mathrm{c}}$ Ref. 33, ${ }^{\mathrm{d}}$ Ref. 34, ${ }^{\mathrm{e}}$ Ref. 35, ${ }^{\mathrm{f}}$ Ref. 36

\section{B. Bandgap and exciton binding energies of $\mathrm{CdX}$ and $\mathrm{HgX}$ NPLs $(\mathrm{X}=\mathrm{Te}, \mathrm{Se})$}

The $E_{G}$ and $E_{B}$ values for the cadmium and mercury NPLs are shown in Table 1 . We note reported values of binding energy in 3-5 ML CdSe NPLs, for which our measurement shows excellent agreement. As expected, the bandgap energy increases as the number of MLs decreases due to increasing quantum confinement. Furthermore, the exciton binding energy also increases as the number of MLs decreases. Interestingly, for the same number of MLs, HgX NPLs have higher $E_{B}$ values (with the exception of $2 \mathrm{ML} \mathrm{CdTe}$ and $\mathrm{HgTe}$ ), which is unexpected as mercury 
chalcogenides have larger dielectric constants than the cadmium ones. ${ }^{37,38}$ This discrepancy may be explained by differences in surface lattice strain between 2 ML CdTe and $\mathrm{HgTe}$, changing valence and conduction band structures and electron-hole interactions. ${ }^{39,40}$ Alternatively, our previous work suggests HgTe NPLs have their outer metal atomic layer stripped off during the cation exchange process, resulting in decreased charge density on the surface and a lower dielectric. ${ }^{10}$ This would potentially result in a larger exciton binding energy in comparison to the metal terminated CdTe NPLs. In the next section we explore how layer number influences the effective dielectric felt by the bound electron-hole pair.

\section{Dielectric Screening}

We hypothesize that exciton binding energies will increase with decreasing NPL thicknesses. To demonstrate this, we model the excitonic interactions in the NPL with an electron and a hole as point charges within a dielectric slab modelled after the dielectric constant of the NPL's $\left(\varepsilon_{N P L} \approx\right.$ 10). This region is sandwiched by an external dielectric equal to that of organic ligand environment $\left(\varepsilon_{e x t} \approx 2\right)$, illustrated in Fig. 3a. We utilize the method of images to determine the excitonic electrostatic potential at one of the charges: ${ }^{41}$

$$
V=\frac{q}{4 \pi \varepsilon_{0} \varepsilon_{N P L} r}+2 \sum_{N=1}^{\infty}\left[\left(\frac{\varepsilon_{N P L}-\varepsilon_{e x t}}{\varepsilon_{N P L}+\varepsilon_{e x t}}\right)^{n} \frac{q}{4 \pi \varepsilon_{0} \varepsilon_{N P L} \sqrt{r^{2}+n^{2} t^{2}}}\right]
$$

where $q$ is the magnitude of the point charge, $\varepsilon_{0}$ is the vacuum permittivity, $\varepsilon_{N P L}$ is the dielectric (i.e. relative permittivity) of the NPL, $\varepsilon_{\text {ext }}$ is the effective dielectric of the external environment, $r$ is the exciton Bohr radius, $t$ is the NPL thickness, and $N$ is the number of image charges (figure 

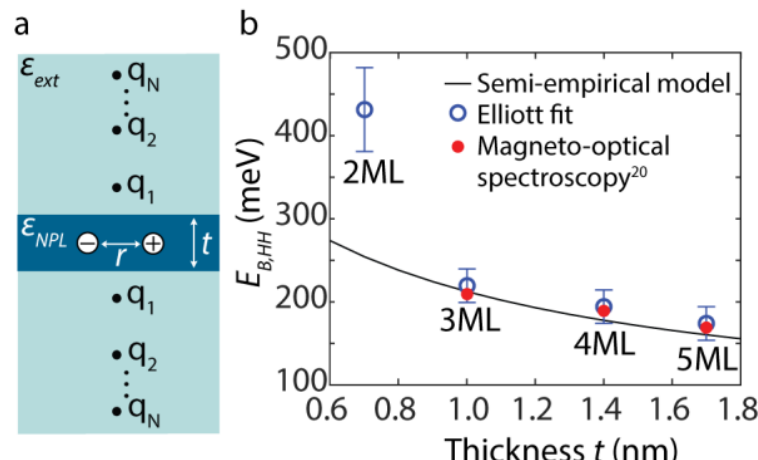

Figure 3. (a) Visualization of the exciton in the colloidal NPL system as represented in the method of images. (b) The relationship between exciton binding energy and CdSe NPL thickness, indicating the predicted trend of our semi-empirical electrostatic model, extracted values from absorption spectra using the modified Elliott model, and measured values from magneto-optical spectroscopy. 3a). The derivation of this potential equation and the effect of $\mathrm{n}$ on the extracted binding energy is explored in SI Sec. VI, where we show $N=20$ image charges reproduce the full potential.

We equate this potential in Eq. 3 to an effective two-point charge Coulombic potential, $V_{e f f}$, with an overall effective relative dielectric constant, $\varepsilon_{\text {eff }}$ :

$$
V_{e f f}=\frac{q}{4 \pi \varepsilon_{0} \varepsilon_{e f f} r}
$$

Solving for $\varepsilon_{e f f}$ allows us to determine the exciton binding energy through the Rydberg equation:

$$
E_{B}=\frac{e^{2}}{8 \pi \varepsilon_{0}^{2} \varepsilon_{e f f}^{2} r}
$$

The Bohr radius value is taken from magneto-optical spectroscopy performed by Brumberg et al. on 3 ML CdSe NPL, which we assume to be constant across all thicknesses. ${ }^{20}$ The $E_{B}$ values for CdSe NPLs of varying thicknesses are shown in Fig. 3b. The electrostatic model captures the trend of decreasing $E_{B}$ with thickness $t$, consistent with our Elliott fit and literature values of $E_{B}$ for 35 ML CdSe NPLs. We note the discrepancy between the fit and model $E_{B}$ at 2 ML, which we attribute to a stronger quantum confinement, resulting in an inaccurate estimation of the Bohr radius used in the model. This discrepancy warrants future investigation with more sophisticated modeling, accounting for wavefunction confinement effects and the interplay between 2D and 3D semiconductors.

\section{Controlling Exciton Binding Energy by Modulating External Dielectric}




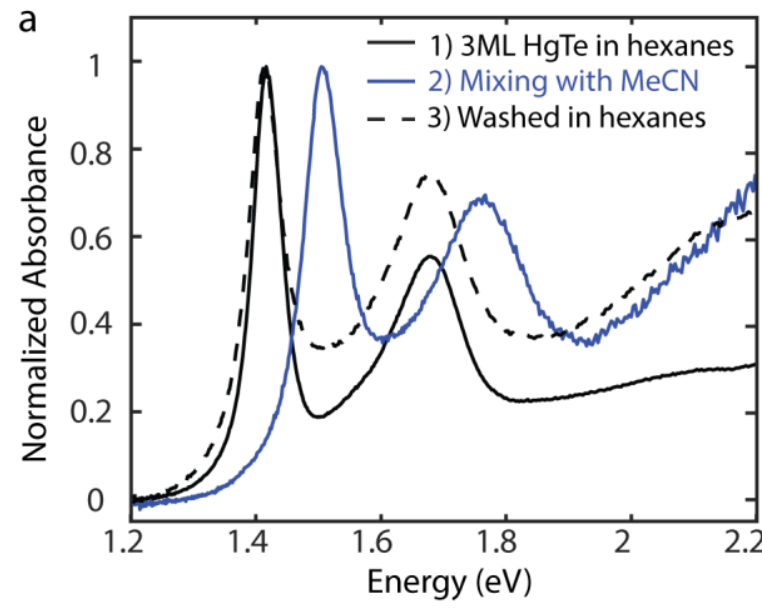

b

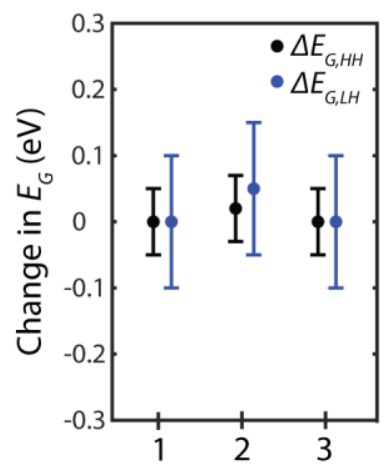

C

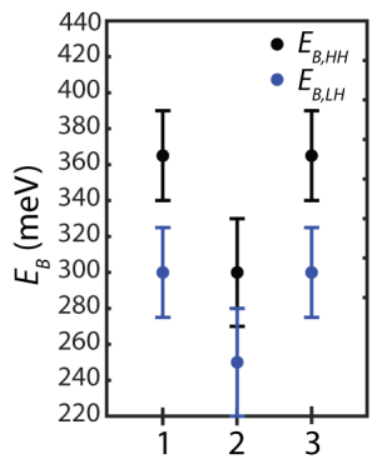

Figure 4. (a) Shift in the $X_{1, H H}$ transition peak as 3 ML HgTe NPLs are vortexed with MeCN mixture and then washed back into hexanes. The baseline increases due to increased Rayleigh scatter. (b) The minimal change in band gap energy as it corresponds to 1 . suspension in hexanes, 2. mixing with $\mathrm{MeCN}$, and 3. washing in hexanes steps, as indicated in (a). (c) The exciton binding energy as it corresponds to steps 1-3, showing a significant decrease in $E_{B}$ with constant $E_{G}$ as the NPLs experience a higher solvent dielectric in step 2 .

To confirm the extent to which external dielectric contrast modulates exciton binding energy, we change the solvent environment, similar to previous work in TMDCs and 2D perovskites. ${ }^{28,42,43}$ We measure $E_{B}$ and $E_{G}$ using the above Elliott model fit of the absorption spectrum of 3 ML HgTe NPLs (Fig. 4a-1), initially suspended in hexanes $\left(\varepsilon_{\text {Hexanes }} \approx 1.9\right)$. Then, acetonitrile (also known as $\mathrm{MeCN}, \varepsilon_{M e C N} \approx 38$ ) is added to the hexane layer, and the solutions are vortexed for 1 minute. After letting it settle, the hexane layer is taken out and its absorption spectrum is measured (Fig. 4a-2; the exact procedure can be found in SI Sec. VII). Finally, the NPLs are returned to their initial hexane environment, after thoroughly washing away the $\mathrm{MeCN}$, and we observe the recovery of the exciton absorption peak, but with increased Rayleigh scatter baseline (Fig. 4a-3). Altogether, Fig. 4a shows the resultant shift and recovery of the $X_{1, H H}$ peak of the HgTe spectrum as the NPLs are exposed to a higher dielectric environment and returned to hexanes. Figs. $4 \mathrm{~b}$ and $4 \mathrm{c}$ demonstrate corresponding Elliott fit values that indicate an unchanged $E_{G}$ in comparison to a $65 \mathrm{meV}$ decrease in $E_{B, H H}$ and a $50 \mathrm{meV}$ decrease in $E_{B, L H}$, supporting the hypothesis that we have increased the effective dielectric and thus lowered the $E_{B}$ without changing the structure of the NPL. From the 
decreasing $E_{B}$ 's, we calculate the corresponding increase in $\varepsilon_{e f f}$ from 13.8 to 15.1 , due to the $\mathrm{MeCN}$ (Eq. 5). Using Eq. 3, we can infer the increase in $\varepsilon_{\text {ext }}$ from 2.0 to 3.3; this is notable as the dielectric of $\mathrm{MeCN}$ is 38 . The small change in $\varepsilon_{\text {ext }}$ suggests the continued dominance of the aliphatic ligands on the dielectric environment, meaning $\mathrm{MeCN}$ only has limited access to the excitonic field lines. Therefore, the shift in $\mathrm{HgTe} E_{B}$ can be attributed to the change in the effective dielectric — suggesting more significant impact of the external dielectric on NPL excitonic behavior, than previously recognized. However, we note that this procedure did not appear to alter the binding energy in cadmium telluride nanocrystals (SI Sec. VII-C), which we attribute to the apparent cadmium termination and more dense ligand coverage in the cadmium chalcogenide nanocrystals. ${ }^{10}$

\section{Conclusion}

Exciton binding energies are important experimental parameters which govern absorption, emission, and energy and charge transport. Using a modified Elliott model, we extract binding energies for many NPLs — several of them for the first time - which also show excellent agreement to prior limited measurements. This model represents a simple and accessible tool to probe excitonic and continuum transitions from absorption measurements, complementing sophisticated techniques such as magneto-optical spectroscopy or opto-acoustic methods which were previously used. $^{20,44}$

We also explore the effect of dielectric screening on NPL excitons using a simple semiempirical electrostatic model, which produced calculated $E_{B}$ 's that are in excellent agreement to measured binding energies for 3-5 ML CdSe NPLs. The model confirmed that unlike their bulk and OD counterparts, nanoplatelet excitonic electrostatic field lines extend beyond the 
semiconductor itself and are modulated by the external environment. We experimentally confirm the effect of dielectric confinement by modifying NPL external environment and showing a noticeable shift in exciton binding energy, consistent with similar measurements in 2D TMDCs

and perovskites. ${ }^{28,42,44}$ Uncovering the exciton binding energies of NPLs and understanding how external dielectric screening modulates these resonances further establishes the distinct properties of nanoplatelets that distinguish them from their quantum dot counterparts.

\section{ASSOCIATED CONTENT}

Supporting Information Available: Supporting Information for Dielectric Confinement Modulates Semiconductor Nanoplatelet Excitons

\section{AUTHOR INFORMATION}

\section{Corresponding Authors}

Timothy L. Atallah - Department of Chemistry and Biochemistry, University of California Los Angeles, 607 Charles E. Young Drive, Los Angeles, California, 90095-1569, United States; Email: atallaht@denison.edu

Justin R. Caram - Department of Chemistry and Biochemistry, University of California Los Angeles, 607 Charles E. Young Drive, Los Angeles, California, 90095-1569, United States; Email: jcaram@chem.ucla.edu

\section{Authors}

Azmain A. Hossain - Department of Chemistry and Biochemistry, University of California Los Angeles, 607 Charles E. Young Drive, Los Angeles, California, 90095-1569, United States; Email: azu07bruin@g.ucla.edu 
Stephanie M. Tenney - Department of Chemistry and Biochemistry, University of California Los Angeles, 607 Charles E. Young Drive, Los Angeles, California, 90095-1569, United States; Email: stenney@ucla.edu

Xuanheng Tan - Department of Chemistry and Biochemistry, University of California Los Angeles, 607 Charles E. Young Drive, Los Angeles, California, 90095-1569, United States; Email: txh1028@g.ucla.edu

Lauren A. Tan - Department of Chemistry and Biochemistry, University of California Los Angeles, 607 Charles E. Young Drive, Los Angeles, California, 90095-1569, United States; Email: laurietan01@g.ucla.edu

Jonathan J. Foley IV - Department of Chemistry, William Paterson University, 300 Pompton Road, Wayne, New Jersey, 07470, United States; Email: foleyj10@wpunj.edu

\section{ACKNOWLEDGEMENT}

This work received partial support was given by National Science Foundation under Career Grant 1905242. Support was also given by the US Department of Energy, Office of Science, Basic Energy Sciences, under QI grant Award DE-SC0019245. Author A. A. H. acknowledges the Dolores C. Southam Summer Undergraduate Research Fellowship from the UCLA Department of Chemistry and Biochemistry. Author J. J. F. acknowledges support from the ART program at William Paterson University. 


\section{References}

1. Tessier, M. D., B. Mahler, B. Nadal, H. Heuclin, S Pedetti, and B. Dubertret. 2013. "Spectrsocopy of colloidal semiconductor core/shell nanoplatelets with high quantum yield." Nano Letters 13: 3321-3328.

2. Nasilowski, M., B. Mahler, E. Lhuillier, S. Ithurria, and B. Dubertret. 2016. "Twodimensional colloidal nanoscrystals." Chemical Reviews 116: 10934-10982.

3. Guzelturk, B., Y. Kelestemur, M. Olutas, S. Delikanli, and H. V. Demir. 2014. "Amplified spontaneous emission and lasing in colloidal nanoplatelets." ACS Nano 8: 6599-6605.

4. Grim, Q., S. Christodoulou, F. D. Stasio, R. Krahne, R. Cingolani, L. Manna, and I. Moreels. 2014. "Continuous-wave biexciton lasing at room temperature using solutionprocessed quantum wells." Nature Nanotechnology 9: 891-895.

5. Ithurria, S., and B. Dubertret. 2008. "Quasi $2 d$ colloidal CdSe platelets with thickness controlled at the atomic level." Journal of American Chemical Society 130: 16504-16505.

6. Bouet, C., B. Mahler, B. Nadal, B. Abecassis, M. D. Tessier, S. Ithurria, X. Xu, and B. Dubertret. 2013. "Two-dimensional growth of CdSe nanocrystals from nanoplatelets to nanosheets." Chemistry of Materials 25: 639-645.

7. Izquierdo, E., M. Dufour, A. Chu, C. Livache, B. Martinez, D. Amelot, G. Patriarche, N. Lequeux, E. Lhuillier, and S. Ithurria. 2018. "Coupled HgSe colloidal quantum wells through a tunable barrier: A strategy to uncouple optical and transport band gap." Chemistry of Materials 30 (12): 4065-4072.

8. Meerbach, C., C. Wu, S. C. Erwin, Z. Dang, A. Prudnikau, and V. Lesnyak. 2020. "Halide-Assisted Synthesis of Cadmium Chalcogenide Nanoplatelets." Chemistry of Materials 32 (1): 566-574.

9. Moghaddam, N., C. Dabard, M. Dufour, H. Po, X. Xu, T. Pons, E. Lhuillier, and S. Ithurria. 2021. "Surface Modification of CdE (E: S, Se, and Te) Nanoplatelets to Reach Thicker Nanoplatelets and Homostructures with Confinement-Induced Intraparticle Type I Energy Level Alignment." Journal of the American Chemical Society 143 (4): 18631872.

10. Tenney, S. M., V. Vilchez, M. L. Sonnleitner, C. Huang, H. C. Friedman, A. J. Shin, T. L. Atallah, A. P. Deshmukh, S. Ithurria, and J. R. Caram. 2020. "Mercury Chalcogenide Nanoplatelet-Quantum Dot Heterostructures as a New Class of Continuously Tunable Bright Shortwave Infrared Emitters." J. Phys. Chem. Lett. 11: 3473-3480. https://doi.org/10.1021/acs.jpclett.0c00958.

11. Hines, M. A., and P. Guyot-Sionnest. 1996. "Synthesis and characterization of strongly luminescing ZnS-capped CdSe nanocrystals." Journal of Physical Chemistry 100: 468471. 
12. Mahler, B., B. Nadal, C. Bouet, G. Patriarche, and B. Dubertret. 2012. "Core/shell colloidal semiconductor nanoplatelets." Journal of American Chemical Society 134: 18591-18598.

13. Christodoulou, S., J. I. Climente, J. Planelles, R. Brescia, M. Prato, B. Martin-Garcia, A. H. Khan, and I. Moreels. 2018. "Chloride-Induced Thickness Control in CdSe Nanoplatelets." Nano Letters 18 (10): 6248-6254.

14. Ithurria, S., G. Bousquet, and B. Dubertret. 2011. "Continuous Transition from 3D to 1D Confinement Observed during the Formation of CdSe Nanoplatelets." Journal of the American Chemical Society 133 (9): 3070-3077.

15. Sun, H., and W. E. Buhro. 2019. "Core-shell cadmoim telluride quantum platelets with absorptions spanning the visible spectrum." ACS Nano 13 (6): 6982-6991.

16. Chaves, J., M. Ribeiro, T. Frederico, and N. M. R. Peres. 2017. "Excitonic effects in the optical properties of $2 \mathrm{~d}$ materials: an equation of motion approach." $2 D$ Materials 4 : 025086.

17. Donck, V. D., and F. M. Peeters. 2018. "Interlayer excitons in transition metal dichalcogenide heterostructures." Physical Review B 98: 115104.

18. Wannier, H. 1937. "The structure of electronic excitation levels in insulating crystals." Physical Review 52: 191-197.

19. Elliott, J. 1957. "Intensity of optical absorption by excitons." Physical Review 108: 13841389.

20. Brumberg, A., S. M. Harvey, J. P. Philbin, B. T. Diroll, B. Lee, S. A. Crooker, M. R. Wasielewski, E. Rabani, and R. D. Schaller. 2019. "Determination of the in-plane exciton radius in 2d CdSe nanoplatelets via magneto-optical spectrocopy." ACS Nano 13: 85898596.

21. Scott, R., A. W. Achstein, A. V. Prudnikau, A. antanovich, L. D. A. Siebbeles, M. Artemyev, and U. Woggon. 2016. "Time-Resolved Stark Spectroscopy in CdSe Nanoplatelets: Exciton Binding Energy, Polarizability, and Field-Dependent Radiative Rates." Nano Letters 16 (10): 6576-6583.

22. Chhowalla, M., D. Jena, and H. Zhang. 2016. "Two-dimensional semiconducors for transistors." Nature Reviews Materials 1: 16052.

23. Ruf, F., M. F. Ayguler, N. Giesbrecht, B. Rendenbach, A. Magin, P. Docampo, H. Kalt, and M. Hetterich. 2019. "Temperature-dependent studies of exciton binding energy and phase-transition suppression in (Cs, FA, MA) $\mathrm{Pb}(\mathrm{I}, \mathrm{Br}) 3$ perovskites." APL Materials 7 : 031113.

24. Green, M. A., A. Ho-Baillie, and H. J. Snaith. 2014. "The emergence of perovskite solar cells." Nature Photonics 8: 506-514. 
25. Blakemore, S. 1982. "Semiconducting and other major properties of gallium arsenide." Journal of Applied Physics 53: R123.

26. Peter, G., E. O. Gobel, P. Dawson, K. Moore, C. Foxon, and R. J. Elliott. 1988. "Linewidth dependence of radiative exciton lifetimes in quantum wells." Physical Review Letters 60 (243).

27. Manser, J. S., J. A. Christians, and P. V. Kamat. 2016. "Intriguing optoelectronic properties of metal halide perovskites." Chemical Reviews 116 (21): 12956-13008.

28. Passarelli, J. V., C. M. Mauck, S. W. Winslow, C. F. Perkinson, J. C. Bard, H. Sai, K. W. Williams, et al. 2020. "Tunable exciton binding energy in $2 \mathrm{~d}$ hybrid layered perovskites through donor-acceptor interactions within the organic layer." Nature Chemistry 12: 672682.

29. Vasiliev, R. B., A. I. Lebedev, E. P. Lazareva, N. N. Shlenskaya, V. B. Zaytsev, A. G., Yao, Y. Vitukhnovsky, and K. Sakoda. 2017. "High-energy exciton transitions in quasi two-dimensional cadmium chalcogenide nanoplatelets." Physical Review B 95: 165414.

30. Moghaddam, N., C. Greboyal, J. Qu, A. Chu, P. Rastogi, C. Livache, A. Kgalili, et al. 2020. "The strong confinement regime in HgTe two-dimensional nanoplatelets." Journal of Physical Chemistry C 124 (42): 23460-23468.

31. Fernandez, M., P. Prete, N. Lovergine, A. Mancini, R. Cingolani, and L. Vasanelli. 1997. "Optical properties of MOVPE-grown ZnS epilayers on (100)GaAs." Physical Review B 55: 7660.

32. Campi, D., and C. Coriasso. 1995. "Optical nonlinearities in multiple quantum wells: Generalized elliott formula." Physical Review B 51: 10719-10728.

33. Soloviev, V. N., Eichhofer, A., Fenske, D., \& Banin, U. (2000). Molecular Limit of a Bulk Semiconductor: Size Dependence of the "Band Gap" in CdSe Cluster Molecules. Journal of American Chemical Society, 122(11), 2673-2674.

34. Kumar, M. M., \& Devadason, S. (2013). Structural and optical properties of CdTe/CdSe heterostructure multilayer thin films prepared by physical vapor deposition technique. Applied Nanoscience, 3, 453-459.

35. Akinlami, J. O., \& Odeyemi, O. O. (2018). Electronic structure and optical properties of HgSe. Semicondcutor Physics, Qhatm Electronics, and Optoelectronics, 21(3), 288-293.

36. Livache, C., Goubet, N., Greboval, C., Martinez, B., Ramade, J., Qu, J., . . Lhuillier, E. (2019). Effect of Pressure on Interband and Intraband Transition of Mercury Chalcogenides Quantum Dots. Journal of Physical Chemistry C, 123(20), 13122-13130.

37. Baars, J., and F. Sorger. 1972. "Reststrahlen spectra of HgTe and CdxHg1-xTe." Solid State Communications 10 (9): 875-878. 
38. Geick, R., C. H. Perry, and S. S. Mitra. 1966. "Lattice vibrational properties of hexagonal CdSe." Journal of Applied Physics 37: 1994-1997.

39. He, K., C. Poole, K. F. Mak, and J. Shan. 2013. "Experimental demonstration of continuous electronic structure tuning via strain in atomically thin MoS2." Nano Letters 13 (6): 2931-2936.

40. Peelaers, H., and C. G. V. de Walle. 2012. "Effects of strain on band structure and effective masses in MoS2." Physical Review B 86: 241401.

41. Jackson, J. D. 1998. Classical Electrodynamics. 3rd. Wiley.

42. Lin, Y., X. Ling, K. Yu, S. Huang, A. L. Hsu, Y. H. Lee, J. Kong, M. S. Dresselhaus, and T. Palacios. 2014. "Dielectric screening of excitons and trions in single-layer MoS2." Nano Letters 14 (10): 5569-5576.

43. Choi, J., H. Zhang, H. Du, and J. H. Choi. 2016. "Understanding solvent effects on the properties of two-dimensional transition metal dichalcogenides." ACS Applied Materials and Interfaces 8 (14): 8864-8869.

44. Zelewski, S. J., K. C. Nawrot, A. Zak, M. Gladysiewicz, M. Nyk, and R. Kudrawiec. 2019. "Exciton binding energy of two-dimensional highly luminescent colloidal nanostructures determine from combined optical and photoacoustic spectroscopies." Journal of Physical Chemistry 10 (12): 3459-3464. 